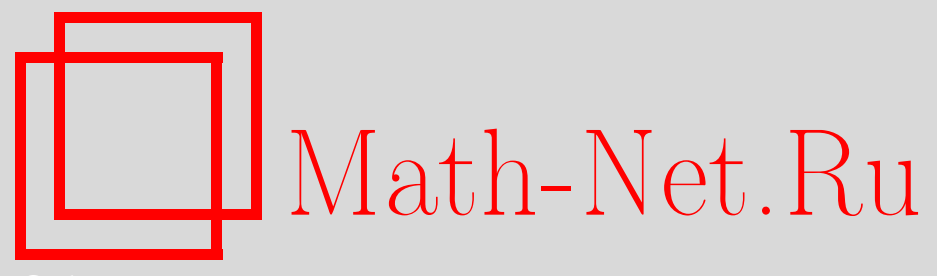

В. А. Щербакова, Классы ориентированных градуированных графов с полиномиально разрешимой мощностной задачей Штейнера, Дискрет. матем., 1997, том 9, выпуск 4, 73-85

DOI: https://doi.org/10.4213/dm503

Использование Общероссийского математического портала Math-Net.Ru подразумевает, что вы прочитали и согласны с пользовательским соглашением http://www.mathnet.ru/rus/agreement

Параметры загрузки:

IP: 34.239 .49 .27

26 апреля 2023 г., 17:06:56 


\title{
Классы ориентированных градуированных графов с полиномиально разрешимой мошностной задачей Штейнера
}

\author{
(C) 1997 г. В. А. Щербакова
}

Рассматривается задача Штейнера на ориентированных градуированных графах, где стоимость всех дуг единична. Дается описание двух классов графов, в которых рассматриваемая задача разрешима за полиномиальное время. Графы из этих классов получаются из деревьев добавлением вершин и ребер, образующих пути длины 2, с сохранением градуированности и достижимости. Предлагаются алгоритмы решения мощностной задачи Штейнера на графах из описанных классов и алгоритмы распознавания принадлежности произвольного ориентированного градуированного графа этим классам. Сложность этих алгоритмов есть $O\left(n^{4}\right)$, где $n-$ число вершин в графе.

\section{1. Введение}

В данной статье рассматривается мощностная задача Штейнера на ориентированном градуированном графе:

для заданного ориентированного, связного по корню $r$ (каждая вершина $v$ в $G$ достижима из корня $r$ ), градуированного (длины всех путей из $r$ в $v$ одинаковы) графа $G=(V, E)$ и подмножества $H \subseteq V$ вершин графа $G$, называемых помеченными, найти дерево Штейнера $T=\left(V^{\prime}, E^{\prime}\right)$, т.е. поддерево графа $G$, связное по корню $r$, содержащее все помеченные вершины, такое, что мощность $\left|E^{\prime}\right|$ минимальна.

Эта задача, являющаяся частным случаем NP-трудной задачи Штейнера на ориентированном графе, остается NP-трудной, что, в частности, показано в [1]. Поэтому представляет интерес проблема поиска таких классов графов, на которых рассматриваемая задача была бы полиномиально разрешимой.

Известно, что задача Штейнера на ориентированном графе полиномиально разрешима в классе последовательно-параллельных графов [2]. В [2] высказано предположение, что это верно и в случае ориентированных последовательнопараллельных графов (т.е. ориентированных графов, которые при отбрасывании ориентации становятся последовательно-параллельными).

В данной статье, в разделах 3 и 4, дается описание двух классов ориентированных градуированных графов, в которых, при подходящем расположении помеченных вершин, мощностная задача Штейнера полиномиально разрешима. Для 
каждого из этих классов графов предлагается алгоритм распознавания принадлежности произвольного ориентированного градуированного графа указанному классу, а также алгоритм решения мощностной задачи Штейнера. Сложность построенных алгоритмов полиномиально зависит от размера графа.

Далее используются следующие обозначения: $p(u, v)$ - путь из $u$ в $v, \rho(u, v)$ - расстояние от $u$ до $v$, т.е. число ребер в пути из $u$ в $v, \operatorname{In}(v)$ - множество всех заходящих в $v$ вершин, т.е. множество вершин, являющихся концами входящих в $v$ peбер, $\operatorname{Out}(v)-$ множество всех исходящих из $v$ вершин, т.е. множество вершин, являющихся концами исходящих из $v$ ребер, $V(G)$ - множество вершин графа $G$, $E(G)$ - множество ребер графа $G, A \hookleftarrow B$ обозначает процедуру переноса элементов множества $B$ в множество $A, V_{i}(G)$ - множество вершин $i$-го уровня графа $G$, т.e.

$$
V_{i}(G)=\{v \in G \mid \rho(r, v)=i\}
$$

\section{2. Задача Штейнера на ориентированном графе}

Если ориентированный градуированный граф $G$ является деревом (т.е. при отбрасывании ориентации граф $G$ не имеет циклов), то каждая вершина в $G$, кроме корня $r$, имеет только одну входящую вершину, где входящей (соответственно, исходящей) вершиной мы называем второй конец входящего (исходящего) ребра. Следовательно, для каждой помеченной вершины заходящая в нее вершина обязана лежать в дереве Штейнера.

Таким образом, получаем простой алгоритм построения дерева Штейнера:

\section{procedure STEINER_TREE;}

begin

$$
\begin{aligned}
& H \hookleftarrow V(T) ; \\
& \text { for } i:=\text { максимальный уровень to } 1 \text { do } \\
& \quad \text { for } v \in V_{i}(G) \text { do } \\
& \quad \text { if } v \in V(T) \text { then } \\
& \quad \operatorname{In}(v) \longleftarrow V(T) ; \\
& E(T):=E(G) \cap(V(T) \times(V(T)) ;
\end{aligned}
$$

end.

Очевидно, что сложность этого алгоритма есть $O(|V|)$ при условии, что хранится список $\operatorname{In}(v)$ для каждой вершины $v$.

Для произвольного ориентированного градуированного графа $G$, если вычислены $\operatorname{In}(v)$, алгоритм распознавания принадлежности графа классу деревьев состоит в подсчете числа вершин в $\operatorname{In}(v)$ для всех $v \in G \backslash\{r\}$. Если $|\operatorname{In}(v)|=1$ для всех $v \neq r$, то $G$ - дерево, в противном случае это не так. Этот алгоритм также линеен.

\section{3. Класс $T P_{2}$}

Графы из класса $T P_{2}$ получаются из ориентированных деревьев добавлением путей длины 2 с сохранением градуированности.

Определение 1. Ориентированное дерево с корнем $r$ принадлежит классу $T P_{2}$. 


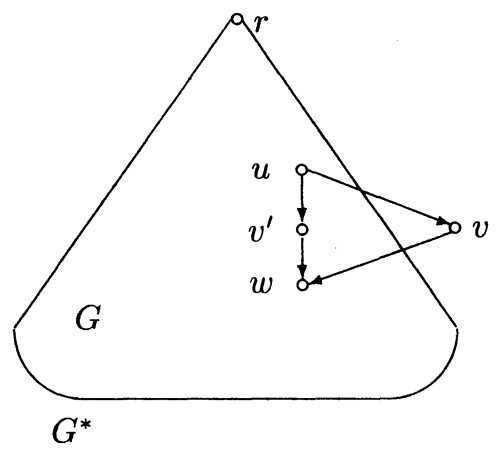

Pис. 1.

Если $G \in T P_{2}$, то граф

$$
G^{*}=(V \cup\{v\}, E \cup\{(u, v),(v, w)\}),
$$

где $u, w \in V(G), v \notin V(G), \rho(u, v)=2$ в графе $G$, тоже принадлежит классу $T P_{2}$ (см. рис. 1).

Класс $T P_{2}$ является подклассом ориентированных градуированных графов, так как любое ориентированное дерево является градуированным графом, а добавление пути сохраняет градуированность. Из определения 1 также следует, что класс $T P_{2}$ содержит в себе класс всех ориентированных деревьев.

Определение 2. Вершина $v$ в графе $G$ удовлетворяет правилу 1 , если ее полустепени захода и исхода равны единице и существует такая вершина $v^{\prime}$, что $\operatorname{In}\left(v^{\prime}\right) \supseteq \operatorname{In}(v)$ and $\operatorname{Out}\left(v^{\prime}\right) \supseteq \operatorname{Out}(v)$.

Лемма 1. Если $G \in T P_{2}$, то, удаляя все вершины, удовлетворяющие правилу 1, пока такие существуют, получим ориентированное дерево с одним корнем.

Доказательство. Граф $G$ получен из ориентированного дерева $T$ добавлением вершин $A=\left\{a_{1}, \ldots, a_{k}\right\}$. Пусть, удалив вершины $B=\left\{b_{1}, \ldots, b_{m}\right\}$, удовлетворяющие правилу 1 , получим граф $G^{*}$, не являющийся деревом.

Пусть существует $b_{i}$ - первая в $B$ вершина, не лежащая в $A$. Поскольку она удовлетворяет правилу 1 она лежит в цикле $\left\{u, v, b_{i}, w\right\}$, где $u$ - заходящая в $v$ и $b_{i}$ вершина, $w$ - общая для $v$ и $b_{i}$ исходящая вершина. Поскольку $b_{i} \in T$ и полустепени захода и исхода $b_{i}$ равны единице, справедливы включения $u, w \in T$. Тогда все остальные вершины, исходящие из $u$ и заходящие в $w$, должны лежать в A.

Если хотя бы одна из этих вершин имеет полустепени захода и исхода в графе $G \backslash\left\{b_{1}, \ldots, b_{i-1}\right\}$, равные единице, то она удовлетворяет правилу 1 . Следовательно, можно в $B$ заменить $b_{i}$ на новую вершину, лежащую в $A$. Если такой вершины нет, то заменим в $T$ вершину $b_{i}$ на любую вершину $a_{j}$, исходящую из $u$ и входящую в $w$, соответственно в $A$ заменим $a_{i}$ на $b_{i}$. 


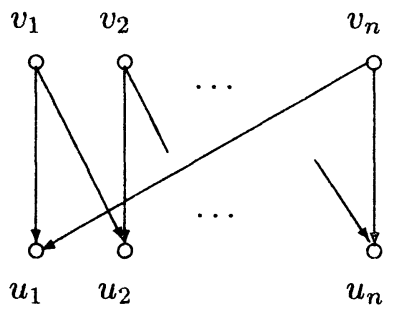

Рис, 2.

Проведя такие замены для всех возможных вершин, получим, что граф $G$ построен из дерева $T^{*}$ добавлением вершин $A^{*}=\left\{a_{1}, \ldots, a_{k}\right\}$. Удалив из $G$ вершины $B^{*} \subseteq A^{*}, B^{*}=\left\{b_{1}, \ldots, b_{m}\right\}$, получим граф $G^{*}$, не являющийся деревом.

Очевидно, что $T^{*} \subseteq G^{*}$. Рассмотрим вершину $a_{j} \in G^{*}, a_{j} \notin B^{*}$, с наибольшим индексом $j$ в $A^{*}$. Поскольку $\left\{a_{j+1}, \ldots, a_{k}\right\} \subseteq B^{*}$, справедливо соотношение $\left\{a_{j+1}, \ldots, a_{k}\right\} \cap G^{*}=\varnothing$. Следовательно, полустепени захода и исхода $a_{j}$ в $G^{*}$ равны единице. Так как $a_{j}$ была добавлена в соответствии с определением 1 , существует вершина $v^{\prime} \in G^{*}$ такая, что у $v^{\prime}$ и $a_{j}$ есть общая заходящая и общая исходящая вершины. Тогда $a_{j}$ удовлетворяет правилу 1 , и мы получаем противоречие.

Следовательно, $G^{*}$ - дерево с тем же корнем $r$, что и граф $G$. Лемма 1 доказана.

Определение 3. Короной длины $n$ будем называть граф $G=(V, E)$, где

$$
\begin{aligned}
& V=\left\{v_{1}, \ldots, v_{n}\right\} \cup\left\{u_{1}, \ldots, u_{n}\right\} \\
& E=\left\{\left(v_{i}, u_{i}\right),\left(v_{i}, u_{(i+1)(\bmod n)}\right) \mid i=1, \ldots, n\right\}
\end{aligned}
$$

(см. рис. 2).

Определение 4. В графе будем называть $M$-конфигурацией пять вершин $\left\{v_{1}, v_{2}, u_{1}, u_{2}, u_{3}\right\}$ таких, что существуют пути

$$
p\left(v_{1}, u_{1}\right), \quad p\left(v_{1}, u_{2}\right), \quad p\left(v_{2}, u_{2}\right), \quad p\left(v_{2}, u_{3}\right)
$$

и не существует путей

$$
p\left(v_{1}, v_{2}\right), \quad p\left(v_{1}, u_{3}\right), \quad p\left(v_{2}, u_{1}\right)
$$

Вершины $u_{1}, u_{3}$ будем называть крайними в $M$-конфигурации, а вершину $u_{2}$ средней (см. рис. 3 ).

Теорема 1. Граф $G \in T P_{2}$ тогда и толъко тогда, когда $G$ удовлетворлет следующиим условиям.

(1) Граф $G$ - ориентированный градуированный граф, связный по корню $r$.

(2) В каждом иикле длины, болъшей четырех, любое ребро принадлежит ииклу длины четыре. 


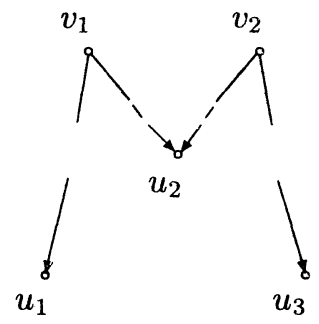

Pис. 3.

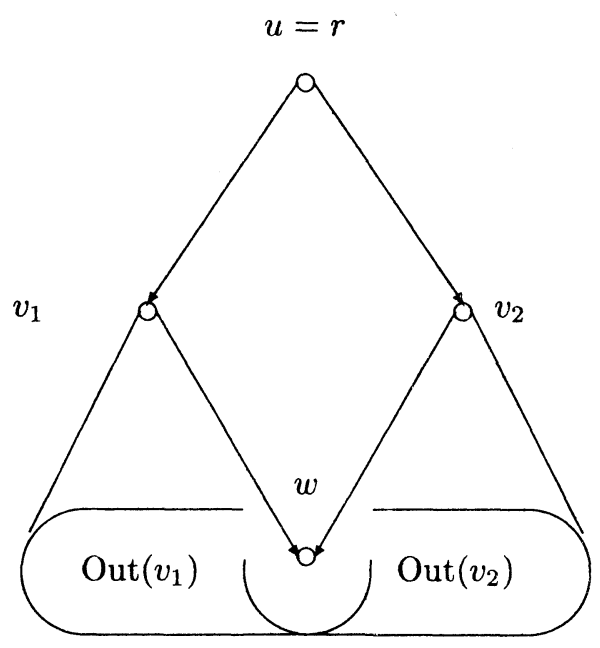

Pис. 4.

(3) В графе $G$ нет подграфов-корон длины $n$ для любого $n \geqslant 2$.

(4) $B$ графе $G$ нет $M$-конфигураций с висячими в $G$ крайними вершинами.

Лемма 2. Если граф $G$ удовлетворяет условиям 1-4 теоремы 1, то либо $G$ является ориентированным деревом с одним корнем $r$, либо существует вершина $v \in G$, удовлетворяющая правилу 1 .

Доказательство. Пусть $G$ удовлетворяет условиям 1-4, но не является деревом и не существует вершины, удовлетворяющей правилу 1.

Проведем доказательство индукцией по количеству уровней графа $G$.

Пусть в $G$ три уровня. Рассмотрим цикл $\left\{u, v_{1}, v_{2}, w\right\}$ (см. рис. 4). При этом $u=r$. Из условия 1 следует, что $\operatorname{In}\left(v_{1}\right)=r=\operatorname{In}\left(v_{2}\right)$.

$\operatorname{Ecли~}\left|\operatorname{Out}\left(v_{1}\right)\right|=1$ (или $\left|\operatorname{Out}\left(v_{2}\right)\right|=1$ ), то получим вершину $v_{1}$ (или $v_{2}$ ), удовлетворяющую правилу 1 , т.е. получим противоречие. Следовательно, $\left|\operatorname{Out}\left(v_{1}\right)\right|>1$, $\left|\operatorname{Out}\left(v_{2}\right)\right|>1$. 
Если $\operatorname{Out}\left(v_{1}\right) \subseteq \operatorname{Out}\left(v_{2}\right)$ (или $\left.\operatorname{Out}\left(v_{2}\right) \subseteq \operatorname{Out}\left(v_{1}\right)\right)$, то получим граф $K_{2,2}$, являющийся короной длины 2.

Если существует $w_{1} \neq w, w_{1} \in \operatorname{Out}\left(v_{1}\right), w_{1} \notin \operatorname{Out}\left(v_{2}\right)$, и существует $w_{2} \neq w, w_{2} \in$ $\operatorname{Out}\left(v_{2}\right), w_{2} \notin \operatorname{Out}\left(v_{1}\right)$, то получим $M$-конфигурацию $\left\{v_{1}, v_{2}, w_{1}, w, w_{2}\right\}$ с висячими крайними вершинами, т.е. получим противоречие.

Следовательно, либо $G$ - дерево, либо есть вершина, удовлетворяющая правилу 1.

Пусть лемма 2 верна для всех графов с $l-1$ уровнем вершин, где $l>3$. Пусть в графе $G$ имеется $l$ уровней.

Из предположения, что $G$ не является деревом, и из условия 2 следует, что существует цикл $\left\{u, v_{1}, v_{2}, w\right\}$ такой, что $v_{1}$ и $v_{2}$ лежат на одном уровне, $u$ - общая заходящая вершина для $v_{1}$ и $v_{2}$, а $w$ - общая исходящая вершина для $v_{1}$ и $v_{2}$, лежащая на наибольшем уровне. Рассмотрим все возможные случаи.

Случай 1. Вершина $w$ лежит не на последнем уровне (т.е. нет таких циклов, в которых $w$ лежала бы на последнем уровне). Тогда, удалив все вершины последнего уровня, получим граф $G^{*}$ с $l-1$ уровнем, удовлетворяющий условиям 1-3 теоремы 1.

Если в $G^{*}$ есть $M$-конфигурация с висячими крайними вершинами, то, добавив к крайним вершинам по одной исходящей вершине последнего уровня, из числа удаленных из $G$, получим $M$-конфигурацию с висячими крайними вершинами в $G$, т.е. получим противоречие. Следовательно, $G^{*}$ удовлетворяет условию 4.

По предположению индукции либо $G^{*}$ - дерево, тогда $G$ - тоже дерево, либо в $G^{*}$ существует вершина, удовлетворяющая правилу 1 , тогда эта же вершина есть и в $G$.

Случай 2. Вершина $w$ лежит в последнем уровне. Построим граф $G^{*}$, отбросив в $G$ корень $r$ и заменив все вершины первого уровня новым корнем $r^{*}$.

Очевидно, что $G^{*}$ удовлетворяет условиям $1-4$ и не является деревом. Следовательно, существует вершина $v$, удовлетворяющая правилу 1 . Если $v$ лежит в $G^{*}$ не на первом уровне, то эта же вершина удовлетворяет правилу 1 и в графе $G$.

Пусть вершина $v$ лежит в $G^{*}$ на первом уровне и не удовлетворяет правилу 1 в графе $G$. Тогда в $G$ вершина $v$ имеет полустепень исхода, равную единице, а полустепень захода, большую единицы.

(a) Вершина $v^{\prime}$, у которой с $v$ есть общая исходящая вершина $c$, имеет полустепень исхода, равную единице (рис.5). Для того, чтобы $v^{\prime}$ не удовлетворяля правилу 1 , должна существовать входящая в $v^{\prime}$ вершина $a_{2}$ такая, что $a_{2} \notin \ln (v)$. Вершины $a_{1}$ и $a_{2}$ имеют только одну входящую вершину, корень $r$. Чтобы они не удовлетворяли правилу 1 , должны существовать вершины $b_{1}$ и $b_{2}$ такие, что

$$
b_{1} \in \operatorname{Out}\left(a_{1}\right), \quad b_{1} \notin \operatorname{Out}\left(a_{2}\right), \quad b_{2} \in \operatorname{Out}\left(a_{2}\right), \quad b_{2} \notin \operatorname{Out}\left(a_{1}\right) .
$$

Из условия 4 следует, что все висячие вершины, достижимые из $b_{1}\left(b_{2}\right)$, должны быть достижимы из $b_{2}\left(b_{1}\right)$ или $c$.

Легко показать, что если существует вершина, достижимая из $b_{1}$ и $b_{2}$, то должна существовать общая исходящая вершина из $b_{1}$ и $b_{2}$. Однако тогда должна существовать и общая заходящая вершина для $b_{1}$ и $b_{2}$, которая будет лежать в короне, соединяющей $b_{1}$ и $b_{2}$. 


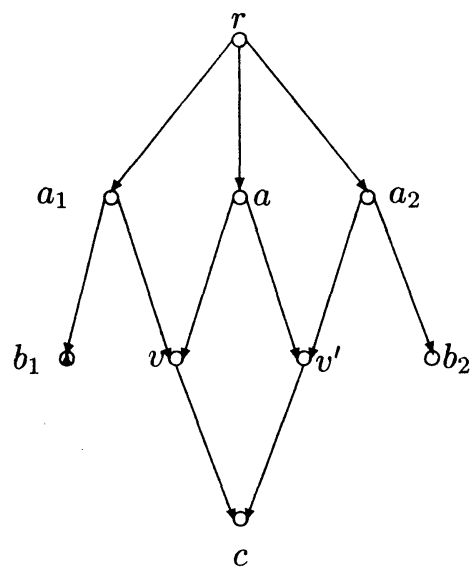

Pис. 5.

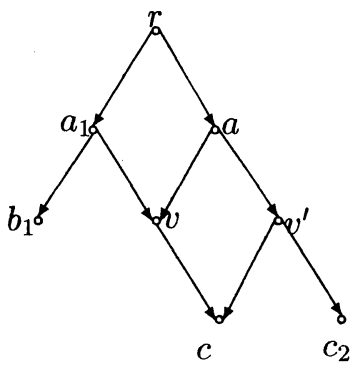

Pис. 6.

Аналогично, если для каждой вершины $c_{i} \in \operatorname{Out}\left(b_{i}\right)$ и $c$ существует общая достижимиая висячая вершина, то существует общая исходящая вершина, а следовательно, общая заходящая вершина, т.е. существует $b_{3} \neq v, b_{3} \neq v^{\prime}$, из которой исходят $c_{i}$ и $c$. В случае $\left|\operatorname{Out}\left(b_{i}\right)\right|>1$ получаем корону. Если $b_{3}=b_{1}$, то пары вершин $b_{1}$ и $v, b_{1}$ и $v^{\prime}$ ддолжны иметь общие заходящие вершины, получаем корону. Если $b_{3} \neq b_{1}$, то пары $b_{3}$ и $v, b_{3}$ и $v^{\prime}, b_{1}$ и $b_{3}$ должны иметь общие заходящие вершины, снова получаем корону.

(b) Полустепень исхода вершины $v^{\prime}$ больше единицы (рис.6). Тогда должны существовать входящая в $v$ вершина $a_{1}$ и исходящая из $v^{\prime}$ вершина $c_{2}$. Для того, чтобы $a_{1}$ не удовлетворяла правилу 1 , должна существовать вершина $b_{1} \neq v, b_{1} \neq v^{\prime}$ исходящая из $a_{1}$.

Из условия 4 следует, что либо всякая висячая вершина, достижимая из $b_{1}$, достижима из $v^{\prime}$, тогда существует корона, соединяющая $b_{1}$ и $v^{\prime}$, либо всякая висячая вершина, достижимая из $c_{2}$, достижима или из $b_{1}$, тогда получаем случай, аналогичный рассмотренному в пункте (a), или из $c$, тогда полустепень исхода вершины $c_{2}$ равна единице. Итак, мы получили вершину $c_{2} \neq v$, аналогичную вершине $v$. Продолжая рассуждения, будем находить новые вершины $c_{i}$, свойства которых 
такие же, как у $v$, что противоречит конечности графа.

Рассмотрев все случаи, мы получали вершину, удовлетворяющую правилу 1 , либо приходили к противоречию. Лемма доказана.

Доказательство теоремъ 1. Пусть $G \in T P_{2}$. Выполнение условий 1 и 2 очевидно следует из определения 1.

Докажем справедливость условия 3. Если в $G$ есть корона длины $n$, то, удалив все выршины, удовлетворяющие правилу 1, мы должны получить дерево. Однако ни одна вершина короны не удовлетворяет правилу 1. Следовательно, корона длины $n$ сохранится после удаления вершин по правилу 1 . Получаем противоречие.

Докажем справедливость условия 4 . Если в $G$ есть $M$-конфигурация, то после удаления вершин по правилу 1 , в графе останется цикл, проходящий через среднюю вершину этой $M$-конфигурации. Получаем противоречие.

Пусть теперь $G$ удовлетворяет условиям 1-4. По лемме 2 либо $G$ - дерево, либо существует вершина $v_{1}$, удовлетворяющая правилу 1 . Очевидно, что граф $G \backslash\left\{v_{1}\right\}$ снова удовлетворяет условиям 1-4.

Выбирая таким образом вершины $\left\{v_{1}, \ldots, v_{k}\right\}$, в конце получим дерево $T=$ $G \backslash\left\{v_{1}, \ldots, v_{k}\right\}$. Следовательно, граф $G$ можно получить из дерева $T$ добавлением вершин $\left\{v_{k}, \ldots, v_{1}\right\}$. По определению 1 такой граф принадлежит $T P_{2}$.

Теорема 1 доказана.

Граф $G$ и множество $H$ помеченных вершин задают мощностную задачу Штейнера из класса $K\left(T P_{2}\right)$, если $G$ принадлежит $T P_{2}$ и $G$ может быть разобран до дерева путем последовательного удаления непомеченных вершин, удовлетворяющих правилу 1. Тогда алгоритм решения задачи из класса $K\left(T P_{2}\right)$ состоит в последовательном поиске и удалении непомеченных вершин, удовлетворяющих правилу 1 , и поиске дерева Штейнера в полученном дереве.

Временная сложность алгоритма сведения графа $G \in T P_{2}$ к дереву составляет $O\left(|V|^{4}\right)$ при условии, что хранятся $\operatorname{In}(v)$ и $\operatorname{Out}(v)$ для каждой вершины графа $G$ и удаление вершины и ребра занимает постоянное время. Поскольку поиск дерева Штейнера на дереве занимает $O(|V|)$ времени, сложность алгоритма поиска дерева Штейнера в классе $K\left(T P_{2}\right)$ составляет $O\left(|V|^{4}\right)$.

Распознавание принадлежности произвольного ориентированного градуированного графа классу $T P_{2}$ состоит из процедуры удаления всех вершин, удовлетворяющих правилу 1 , и проверки принадлежности полученного графа классу деревьев. Сложность этого алгоритма есть также $O\left(|V|^{4}\right)$.

\section{4. Класс $T S_{2}$}

Графы из класса $T S_{2}$ получаются из ориентированных деревьев добавлением ориентированной звезды, т.е. набора ребер

$$
\left(u_{i}, v\right),\left(v, w_{j}\right), \quad i=1, \ldots, k, \quad j=1, \ldots, m .
$$

Определение 5. Ориентированное дерево с корнем $r$ принадлежит классу $T S_{2}$.

Если $G \in T S_{2}$, то граф

$$
G^{*}=(V \cup\{v\}, E \cup\{(u, v) \mid u \in U\} \cup\{(v, w) \mid w \in W\}),
$$




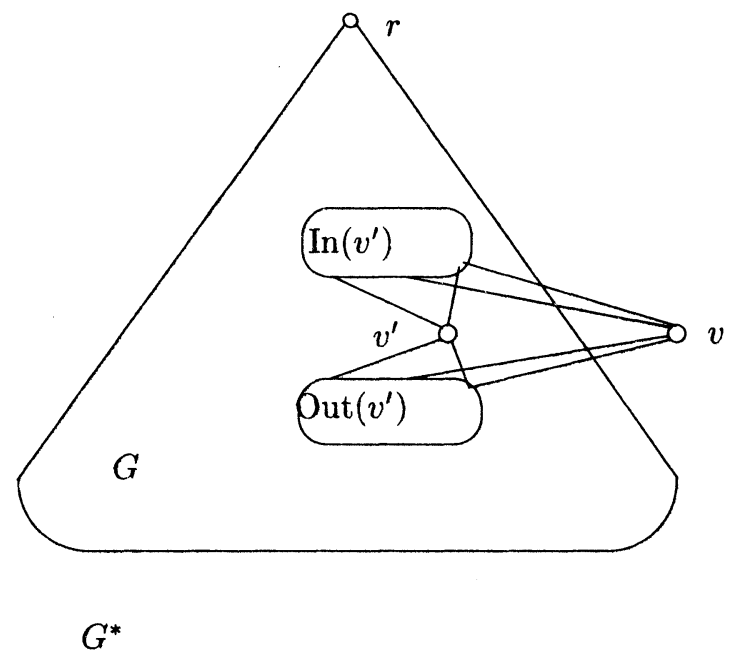

Рис. 7.

где $v \notin V(G), v^{\prime} \in V(G), U \subseteq \operatorname{In}\left(v^{\prime}\right), W \subseteq \operatorname{Out}\left(v^{\prime}\right), U \neq \varnothing, W \neq \varnothing$, тоже принадлежит классу $T S_{2}$ (см. рис. 7).

Очевидно, что класс $T S_{2}$ является подклассом класса ориентированных градуированных графов и содержит в себе класс $T P_{2}$.

Oпределение 6. Вершина $v$ в графе $G$ удовлетворяет правилу 2 , если $|\operatorname{In}(v)|>0$, $|\operatorname{Out}(v)|>0$, и существует вершина $v^{\prime}$ такая, что $\operatorname{In}\left(v^{\prime}\right) \supseteq \operatorname{In}(v)$ и $\operatorname{Out}\left(v^{\prime}\right) \supseteq \operatorname{Out}(v)$.

Лемма 3. Если $G \in T S_{2}$, то, удалив все вершины, удовлетворяющие правилу 2, получим ориентированное дерево с одним корнем.

Доказательство. Граф $G$ получен из ориентированного дерева $T$ добавлением вершин $A=\left\{a_{1}, \ldots, a_{k}\right\}$. Пусть, удалив вершины $B=\left\{b_{1}, \ldots, b_{m}\right\}$, удовлетворяющие правилу 2 , получим граф $G^{*}$, не являющийся деревом.

Пусть существует вершина $b_{i} \in B, b_{i} \notin A$, с наименьшим в $B$ индексом $i$. Поскольку она удовлетворяет правилу 2 , существует вершина $v^{\prime}$ такая, что

$$
\operatorname{In}\left(b_{i}\right) \subseteq \operatorname{In}\left(v^{\prime}\right), \quad \operatorname{Out}\left(b_{i}\right) \subseteq \operatorname{Out}\left(v^{\prime}\right)
$$

Вершина $b_{i} \in T$, следовательно, существуют $u \in \operatorname{In}\left(b_{i}\right)$ и $w \in \operatorname{Out}\left(b_{i}\right), u, w \in T$. Тогда любая вершина, исходящая из $u$ и входящая а $w$, должна лежать в $A$.

Если среди таких вершин есть вершина $a_{j}$ такая, что

$$
\operatorname{In}\left(a_{i}\right) \subseteq \operatorname{In}\left(b_{i}\right), \quad \operatorname{Out}\left(a_{i}\right) \subseteq \operatorname{Out}\left(b_{i}\right)
$$

то заменим в $B$ вершину $b_{i}$ на $a_{j}$.

Если такой вершины нет, то заменим в $T$ вершину $b_{i}$ на любую $a_{j}$, исходящую из $u$ и входящую в $w$, соответственно, в $A$ заменим $a_{j}$ на $b_{i}$. 
Проведя такие замены для всех возможных вершин, получим, что граф $G$ построен из дерева $T^{*}$ добавлением вершин $A^{*}=\left\{a_{1}, \ldots, a_{k}\right\}$. Удалив из $G$ вершины множества $B^{*}=\left\{b_{1}, \ldots, b_{m}\right\}, B^{*} \subseteq A^{*}$, получим граф $G^{*}$, не являющийся деревом.

Очевидно, что $T^{*} \subseteq G^{*}$. Рассмотрим вершину $a_{j} \in G^{*}, a_{j} \notin B^{*}$, с наиюольшим в $A^{*}$ индексом $j$. Поскольку $\left\{a_{j+1}, \ldots, a_{k}\right\} \subseteq B^{*}$, имеем $\left\{a_{j+1}, \ldots, a_{k}\right\} \cap G^{*}=\varnothing$. Следовательно, $\operatorname{In}\left(a_{j}\right)$ и $\operatorname{Out}\left(a_{j}\right)$ в $G^{*}$ такие же, как на момент добавления $a_{j}$, т.е. существует $v^{\prime} \in G^{*}$ такая, что

$$
\operatorname{In}\left(a_{i}\right) \subseteq \operatorname{In}\left(v^{\prime}\right), \quad \operatorname{Out}\left(a_{i}\right) \subseteq \operatorname{Out}\left(v^{\prime}\right)
$$

Таким образом, $a_{j}$ удовлетворяет правилу 2, получаем противоречие.

Лемма 1 доказана.

Теорема 2. Граф $G \in T S_{2}$ тогда и толъко тогда, когда $G$ удовлетворлет следующим условиям.

(1) Граф $G$ - ориентированный градуированный граф, связный по корню $r$.

(2) Если две вершины одного уровня имеют общую исходящую вершину, то у них есть общая заходящая вершина.

(3) В каждом чикле длины, большей четырех, любое ребропринадлежит ииклу длины четыре.

(4) Если в графе $G$ есть $M$-конфигурация с висячими крайними вершинами $v_{1} u$ $v_{2}$, то существует вершина $w$, из которой достижимы средняя и обе крайние вершины $M$-конфигурации и

$$
\rho(r, w) \geqslant \min \left(\rho\left(r, v_{1}\right), \rho\left(r, v_{2}\right)\right)
$$

(c.M. puc.3)

Лемма 4. Если граф $G$ удовлетворяет условиям 1-4 теоремы 2, то либо $G$ является ориентированным деревом с корнем $r$, либо существует вериина $v \in G$, удовлетворяющая правилу 2.

Доказательство. Пусть $G$ удовлетворяет условиям $1-4$, но не является деревом и не существует вершин, удовлетворяющих правилу 2.

Проведем доказательство индукцией по числу уровней в графе $G$.

Пусть в графе $G$ три уровня вершин. Рассмотрим цикл $\left\{u, v_{1}, v_{2}, w\right\}$ (рис.8). При этом $u=r$. Из условия 1 следует, что $\operatorname{In}\left(v_{1}\right)=r=\operatorname{In}\left(v_{2}\right)$.

$\operatorname{Ecли~} \operatorname{Out}\left(v_{1}\right) \notin \operatorname{Out}\left(v_{2}\right)$ и $\operatorname{Out}\left(v_{2}\right) \notin \operatorname{Out}\left(v_{1}\right)$, то имеем $M$-конфигурацию для каждой вершины $u_{1} \in \operatorname{Out}\left(v_{1}\right) \backslash \operatorname{Out}\left(v_{2}\right)$ и каждой вершины $u_{3} \in \operatorname{Out}\left(v_{2}\right) \backslash \operatorname{Out}\left(v_{1}\right)$. По условию 4 для каждой $M$-конфигурации существует вершина $v_{3}$, входящая для $u_{1}, w$, и $u_{3}$.

Если все эти вершины различны, то получим корону длины

$$
n=\left|\operatorname{Out}\left(v_{2}\right) \backslash \operatorname{Out}\left(v_{1}\right)\right|+\left|\operatorname{Out}\left(v_{1}\right) \backslash \operatorname{Out}\left(v_{2}\right)\right| \text {. }
$$

Тогда по условию 3 существует вершина $v^{*}$ такая, что $\operatorname{In}\left(v^{*}\right)=r, \operatorname{Out}\left(v^{*}\right) \supseteq \operatorname{Out}\left(v_{1}\right)$, т.е. $v_{1}$ удовлетворяет правилу 2 . Получаем противоречие. 


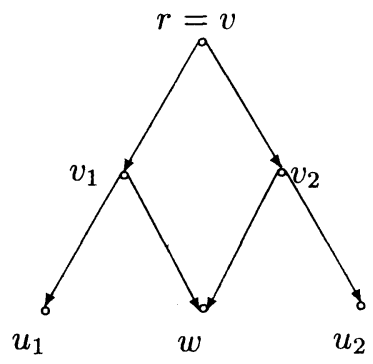

Рис. 8.

Если все вершины $v_{3}$ совпадают, то получим, что $\operatorname{Out}\left(v_{3}\right) \supseteq \operatorname{Out}\left(v_{1}\right)$, т.е. $v_{1}$ удовлетворяет правилу 2.

Если совпадают только некоторые из $v_{3}$, то получим корону длины, меньшей $n$, содержащую все вершины из Out $\left(v_{1}\right)$. Таким образом, $v_{1}$ снова удовлетворяет правилу 2. Получаем противоречие.

Следовательно, либо $G$ - дерево, либо есть вершина, удовлетворяюшая правилу 2 .

Пусть теперь лемма 4 верна для всех графов с $l-1$ уровнем вершин, $l>3$.

Пусть в графе $G$ имеется $l$ уровней. Построим граф $G^{*}$, удалив в $G$ корень $r$ и заменив все вершины первого уровня новым корнем $r^{*}$. Очевидно, что $G^{*}$ имеет $l-1$ уровней и удовлетворяет условиям 1-4. Рассмотрим все возможные случаи.

Случай 1. Пусть $G^{*}$ - дерево. Тогда в $G$ есть циклы вида $\left\{r, v_{1}, v_{2}, w\right\}$, где $v_{1}, v_{2}$ лежат на первом уровне, а $w$ на втором. Кроме того, циклов на других уровнях нет.

$\operatorname{Ecли~} \operatorname{Out}\left(v_{1}\right) \nsubseteq \operatorname{Out}\left(v_{2}\right)$ и $\operatorname{Out}\left(v_{2}\right) \nsubseteq \operatorname{Out}\left(v_{1}\right)$, то имеются такие $M$-конфигурации, что вершины $v_{3}$, из которых достижимы крайние и средняя вершины, могут лежать только на первом уровне. Рассмотрев их, как и в случае графа на трех уровнях, получим вершину, удовлетворяющую правилу 2 , т.е. получим противоречие.

Случай 2. Пусть в $G^{*}$ есть вершина $v$, удовлетворяющая правилу 2 и лежащая не на первом уровне. Тогда эта же вершина будет удовлетворять правилу 2 и в графе $G$. Получаем противоречие.

Случай 3. Пусть в $G^{*}$ есть вершина $u_{2}$, удовлетворяющая правилу 2 и лежащая на первом уровне, т.е. в $G$ есть вершина $u_{1}$ такая, что $\operatorname{Out}\left(u_{2}\right) \subseteq \operatorname{Out}\left(u_{1}\right)$, но $\operatorname{In}\left(u_{2}\right) \nsubseteq$ $\operatorname{In}\left(u_{1}\right)$ (см. рис. 9 ). Поскольку $v_{2}$ не должна удовлетворять правилу 2 , существует $u_{3} \notin \operatorname{Out}\left(v_{1}\right)$.

(a) Пусть в $G$ есть $M$-конфигурация, проходящая через $\left\{v_{1}, v_{2}, u_{1}, u_{2}, u_{3}\right\}$. Тогда есть общая входящая для $u_{2}, u_{1}^{\prime}, u_{3}^{\prime}$ вершина $v_{3}$, где $\left\{v_{1}, v_{2}, u_{1}^{\prime}, u_{2}, u_{3}^{\prime}\right\}$ лежат в некоторой $M$-конфигурации.

Если все вершины из Out $\left(v_{1}\right)$ лежат в какой-нибудь $M$-конфигурации, то из условий 3,4 , и конечности ширины графа получаем, что существует вершина $v_{3}$ такая, что

$$
\operatorname{Out}\left(v_{1}\right) \subseteq \operatorname{Out}\left(v_{3}\right), \quad \operatorname{In}\left(v_{1}\right)=\operatorname{In}\left(v_{2}\right)=r
$$




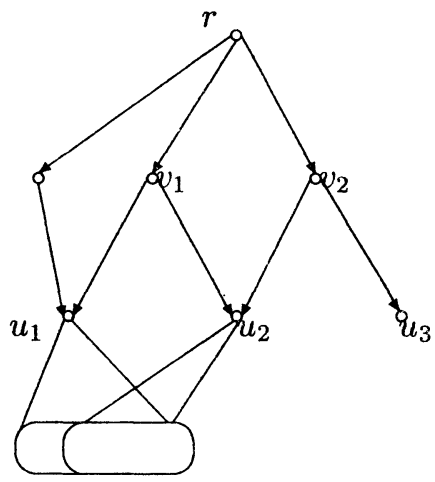

Рис. 9.

т.е. $v_{1}$ удовлетворяет правилу 2. Получаем противоречие.

(b) Вершины $\left\{v_{1}, v_{2}, u_{1}, u_{2}, u_{3}\right\}$ не лежат в $M$-конфигурации из-за достижимости крайних висячих вершин из $v_{1}$ и $v_{2}$. Тогда любая пара вершин $u_{1}, u_{3}^{*}$, где

$$
u_{3}^{*} \in \operatorname{Out}\left(v_{2}\right), \quad u_{3}^{*} \neq u_{2},
$$

имеет общую достижимую вершину, следовательно, общую исходящую и общую заходящую вершины. Это же верно и для любой пары $u_{1}^{*}, u_{3}$, где $u_{1}^{*} \in \operatorname{Out}\left(v_{1}\right)$. Тогда из условия 3 следует, что найдется вершина $v^{*}$ такая, что

$$
\operatorname{Out}\left(v_{1}\right) \subseteq \operatorname{Out}\left(v^{*}\right), \quad \operatorname{In}\left(v^{*}\right)=r
$$

т.е. $v_{1}$ удовлетворяет правилу 2. Получаем противоречие.

(c) Вершины $\left\{v_{1}, v_{2}, u_{1}, u_{2}, u_{3}\right\}$ не лежат в $M$-конфигурации из-за достижимости одной крайней вершины из средней. Тогда любая пара $u_{1}^{*}, u_{2}$, где $u_{1}^{*} \in \operatorname{Out}\left(v_{1}\right)$, $u_{1}^{*} \neq u_{2}$, имеет общую исходящую и общую входящую вершины. Это приводит к тому, что найдется вершина $v^{*}$ такая, что

$$
\operatorname{Out}\left(v_{1}\right) \subseteq \operatorname{Out}\left(v^{*}\right), \quad \operatorname{In}\left(v^{*}\right)=r
$$

Таким образом, мы опять получаем противоречие.

Все случаи привели к противоречию, следовательно, граф $G$ - дерево или в $G$ есть вершина, удовлетворяющая правилу 2. Лемма 4 доказана.

Доказательство теоремъ 2. Пусть $G \in T P_{2}$. Выполнение условий 1 и 2 прямо следует из определения 5.

Докажем справедливость условия 3 . Если в $G$ есть корона длины $n$, то, удалив все вершины, удовлетворяющие правилу 2 , мы должны получить дерево. Для этого нужно, чтобы были удалены все (кроме, быть может, одной) верхние вершины короны и чтобы оставшиеся вершины не имели общих исходящих вершин в короне. Это возможно только тогда, когда все нижние вершины короны имеют общую входящую вершину. 
Докажем справедливость условия 4. Пусть в $G$ есть $M$-конфигурация, но не существует вершины, из которой достижимы крайние и средняя вершины. Тогда средняя вершина и два входящих в нее ребра останутся после удаления всех вершин, удовлетворяющих правилу 2. Получаем противоречие.

Пусть теперь $G$ удовлетворяет условиям $1-4$. По лемме 4 либо $G$ - дерево, либо существует вершина $v_{1}$, удовлетворяющая правилу 2. Очевидно, граф $G \backslash\left\{v_{1}\right\}$ снова удовлетворяет условиям 1-4.

Выбирая таким образом вершины $\left\{v_{1}, \ldots, v_{k}\right\}$, в конце получим дерево $T=$ $G \backslash\left\{v_{1}, \ldots, v_{k}\right\}$. Следовательно, граф $G$ можно получить из дерева $T$ добавлением вершин $\left\{v_{k}, \ldots, v_{1}\right\}$. По определению 5 такой граф принадлежит $T S_{2}$.

Теорема 2 доказана.

Граф $G$ и множество $H$ непомеченных вершин задают мощностную задачу Штейнера из класса $K\left(T S_{2}\right)$, если $G$ принадлежит $T S_{2}$ и $G$ может быть разобран до дерева путем последовательного удаления непомеченных вершин, удовлетворяющих правилу 2. Тогда алгоритм решения задачи из класса $K\left(T S_{2}\right)$ состоит в последовательном поиске и удалении непомеченных вершин, удовлетворяющих правилу 2 , и поиске дерева Штейнера в полученном дереве. Сложность этого алгоритма есть $O\left(|V|^{4}\right)$.

Распознавание принадлежности произвольного ориентированного градуированниго графа классу $T S_{2}$ состоит из процедуры удаления вершин, удовлетворяющих правилу 2 , до тех пор, пока такие вершины существуют, и проверки, является ли полученный граф деревом. Сложность этого алгоритма есть также $O\left(|V|^{4}\right)$.

\section{Список литературы}

1. Щербакова В. А. Минимальные деревья, связывающие заданное подмножество вершин в ориентированном градуированном графе. Деп. ВИНИТИ 21.10.94, №2391-В94.

2. Takamizawa K., Nishizeki T., Saito N. Linear-time computability of combinatorial problems on series-parallel graphs. J. Assoc. Computing Machinery (1982) 29, 623-641.

Статья поступила 08.09.1994. Переработанный вариант поступил 07.09.1997. 